\title{
Efficient Protection Schemes for Hybrid WDM/TDM Passive Optical Networks
}

\author{
Abhishek Dixit, Bart Lannoo, Didier Colle, Mario \\ Pickavet \\ Department of Information Technology \\ Ghent University-IBBT \\ Ghent, Belgium \\ abhishek.dixit@intec.ugent.be
}

\begin{abstract}
Hybrid WDM/TDM Passive Optical Network (PON) is a promising candidate for next-generation optical access (NGOA) solutions as it is able to offer a high splitting ratio and consequently achieves a relatively low cost and power consumption on a per-user basis compared with other NGOA architectures. On the other hand, the end users require a certain level of connection availability while the operators need to reduce the failure impact (i.e. to avoid a huge number of end users being affected by any single failure). Therefore, by evaluating the connection availability and failure impact robustness we identify the most efficient parts to provide resilience in a hybrid WDM/TDM PON from an operator and an end-user perspective. Then, we select the appropriate protection schemes to construct some novel reliable architectures and analyze their reliability performance in urban and rural scenarios. In this way, this paper provides a comprehensive insight into the most relevant protection mechanisms for hybrid WDM/TDM PONs.
\end{abstract}

Keywords-- Resilience, Availability, Failure Impact Robustness, Hybrid WDM/TDM PON

\section{INTRODUCTION}

Node consolidation, where multiple central offices (COs) are being replaced by a single $\mathrm{CO}$ at the central access node (CAN) covering larger service areas, is an on-going trend in optical access network evolution. It is particularly driven by operators for reducing the operational cost of the network. Meanwhile, bandwidth demanding applications, such as highdefinition television (HDTV), real-time interactive gaming, telemedicine, etc. as well as user behavior (always on) are creating a new challenge for efficiently offering high bandwidth on a per-user basis in access networks. Therefore, next-generation optical access (NGOA) networks are expected to support a large service area (i.e. long reach and large splitting ratio), while providing a high sustainable bandwidth for each user. Several wavelength division multiplexing (WDM)-based passive optical network (PON) architectures, possibly complemented by time division multiplexing (TDM) techniques, are able to support the NGOA requirements [1]. Compared with other NGOA architectures, hybrid wavelength and time division multiplexing (WDM/TDM) PON offers a relatively large splitting ratio, and consequently can achieve a lower cost and power consumption per user [2]. Moreover, it inherently supports high flexibility of resource allocation [3] which allows it to efficiently adapt to the varied traffic demands from the end user. Therefore, hybrid WDM/TDM

\author{
Jiajia Chen, Mozhgan Mahloo \\ School of Information and Communication Technology \\ The Royal Institute of Technology KTH \\ Kista, Sweden \\ \{jiajiac, mahloo\}@kth.se
}

PON is considered as a promising candidate for NGOA solutions.

On the other hand, due to the larger coverage and higher bandwidth provision in NGOA networks, fault management becomes more important for a reliable service delivery and business continuance. From the operators' point of view, reducing the failure impact (i.e. to minimize the number of end users affected by a single failure) should be considered in the first place. Meanwhile, the end users (in particular business users) typically require a certain guaranteed level of connection availability in order to lower the risk of service interruption.

With this in mind, this paper focuses on hybrid WDM/TDM PONs and provides a comprehensive insight into the most efficient protection schemes. We take into account two reliability performance parameters, namely, connection availability and failure impact robustness, which can represent an end-user and an operator perspective, respectively. By evaluating these two parameters, we identify the most relevant parts to provide protection in a hybrid WDM/TDM PON. Finally, we combine the selected resilience mechanisms to construct several novel reliable architectures.

The remainder of this paper is organized as follows. Section II describes different variants of hybrid WDM/TDM PON architectures. Section III introduces performance parameters and scenarios considered for the reliability assessment. Section IV presents the evaluation of unprotected variants of hybrid WDM/TDM PON and identifies the most important parts for protection. In Section $\mathrm{V}$, we propose reliable architectures and evaluate them in different population areas and the final section presents our conclusions.

\section{HYBRID WDM/TDM PON ARCHITECTURES}

In this section, different variants of hybrid WDM/TDM PON architectures are described. Typically they have a tree topology, with the optical line terminal (OLT) as the root of the tree and the optical network units (ONUs) at the leaves (see Fig. 1). Fig. 1 shows the detailed system design of the OLT for a 40 upstream (ONU to OLT) and downstream (OLT to ONU) channels configuration. We use photonic integrated circuits (PIC) based transceivers arrays (TRXA) and a series of L and $\mathrm{C}$ band diplexers to multiplex and demultiplex downstream and upstream channels respectively. In a node consolidation scenario, the OLT is located at the CAN which is connected to 


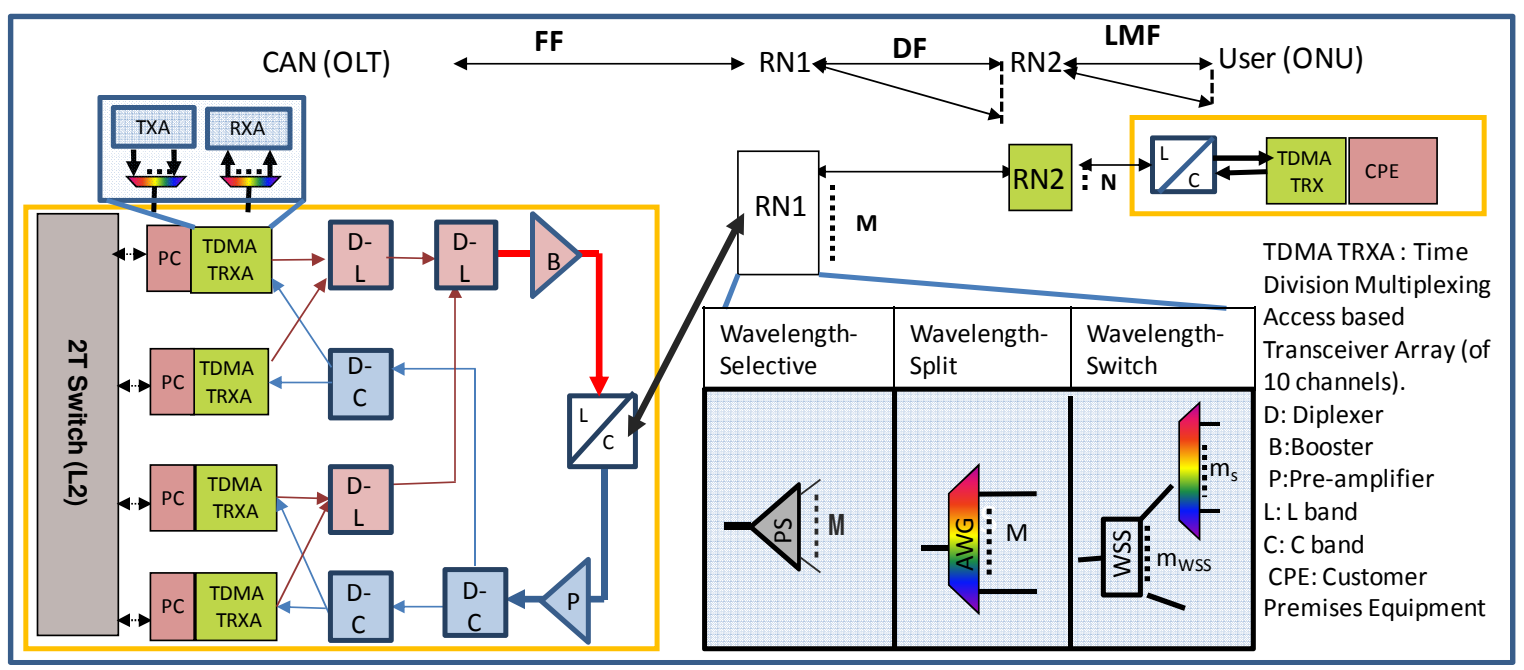

Figure 1. Basic hybrid WDM/TDM PON architecture

remote node 1 (RN1), typically at the local exchange (LE), by the feeder fiber (FF). Through the distribution fiber (DF), each output port of RN1 goes to a different remote node 2 (RN2) which includes a power splitter, and then each output port of the power splitter is connected to a different ONU by the last mile fiber (LMF). According to the RN1 configuration, we have three variants of hybrid WDM/TDM PON architectures. For all variants, we have assumed 80 upstream and downstream wavelength channels.

\section{A. Wavelength Selective Hybrid WDM/TDM PON}

As shown in Fig. 1, RN1 of a wavelength selective hybrid WDM/TDM PON consists of power splitters (PS). As a consequence, this implies a broadcast and select behavior since each ONU has to ultimately select its assigned wavelength and time slot. This approach has the highest flexibility on resource allocation among all hybrid WDM/TDM PON variants, but at the expense of a huge insertion loss occurred by the high power splitting ratio. For this study, a 1:32 splitter has been assumed at both RN1 and RN2 (i.e. $M=N=32$ ). To achieve any kind of reach, the wavelength selective hybrid WDM/TDM PON should always come with a booster (downstream) and a preamplifier (upstream) at the OLT and a reach extender (RE) at $\mathrm{RN} 1$. Note that the reach is limited by the downlink direction, and we have assumed a booster gain of $2.5 \mathrm{~dB}$ for laser class $1 \mathrm{M}$ safety considerations, and a RE gain of $20 \mathrm{~dB}$. Further, the values for the insertion loss of the components are coming from [2].

\section{B. Wavelength Split Hybrid WDM/TDM PON}

A wavelength split hybrid WDM/TDM PON uses arrayed waveguide gratings (AWG) at RN1. In this way, one dedicated wavelength is routed to each RN2. Although this configuration is limited in flexibility on wavelength allocation, it has a relatively long reach due to the low insertion loss of an AWG. We consider a 1:80 AWG at RN1 and a 1:16 splitter at RN2 (i.e. $M=80, N=16$ ). This hybrid WDM/TDM PON can have a reach of $8 \mathrm{~km}$ without booster/pre-amplifier and of $16 \mathrm{~km}$ without RE. We have considered specific configurations of wavelength split hybrid WDM/TDM PON for a required reach.

\section{Wavelength Switched Hybrid WDM/TDM PON}

In wavelength switched hybrid WDM/TDM PON, active optical components requiring a power supply and electronic control like wavelength selective switch (WSS) are installed at RN1. It provides a partial degree of flexible wavelength allocation [3]. For this study, we consider a 1:4 WSS, and a 1:10 AWG at RN1 and a 1:32 splitter at RN2 (i.e. $M=m_{W S S} \times$ $m_{S}=40, N=32$ ). We always consider a RE at RN1, and a booster and pre-amplifier at OLT in order to achieve a sufficient reach.

\section{PERFORMANCE PARAMETERS AND SCENARIOS FOR RELIABILTY EVALUATION}

In this section, we discuss the used reliability performance parameters and the scenarios considered for evaluation.

\section{A. Reliability Performance Parameters}

Two parameters are considered important for a reliability measurement: availability and failure impact robustness.

\section{1) Component and Connection Availability}

Asymptotic availability is defined as the probability that a component is operable at an arbitrary point of time. The approximate equation of availability $A$ [4] for a certain component can be expressed as:

$$
A=1-\frac{M T T R}{M T B F}
$$

with: $M T T R=$ mean time to repair

$$
M T B F=\text { mean time between failures }
$$

MTBF and MTTR values of each element are given in [2]. Connection availability means the probability that a logical connection (e.g. between the OLT and ONU) is operable.

\section{2) Failure Impact Robustness (FIR)}

Besides availability, we consider another important resilience parameter, namely the failure impact robustness (FIR), which is comparable to the figure of merit (FOM) introduced in [5]. The failure of an OLT (at the CAN) impacts all customers whereas the failure of an ONU (at the user end) affects just one customer. In reality, network operators are 
often more worried about a single failure with large impact than many small uncorrelated failures (with the same total impact), since a single large impact failure does more harm to the company image and could lead to negative press releases. Moreover, an operator will feel more economic stresses due to failures with a large impact as it involves a high one-time penalty cost compared to failures with a small impact where the penalty cost is gradual. To reflect this reality, the FIR is a better measure. For a specific component, it is given as:

$$
F I R_{\text {component }}=\frac{1}{C A F \times U n A v}
$$

with: $C A F=$ Number of customers affected by a failure $U n A v=$ Unavailability of the component $=1-\mathrm{A}$

The FIR of the end-to-end (EtoE) connection, consisting of a sequence of components (e.g. OLT, ONU, RN1, RN2) can be evaluated by:

$$
\frac{1}{F I R_{\text {EtoE }}}=\sum \frac{1}{\text { FIR }_{\text {component } i}}
$$

\section{3) Optimal performance parameter value}

The optimal value of the availability and FIR depends on the network operator and the customers in operation. However, we feel that an availability of $>0.9999$ is sufficient for NGOA networks as the aggregation networks were also built with an availability of 4 nines [6].

For a robustly built network (with an availability of 0.9999), a failure in the network with FIR > 10 will affect less than 1000 users at the same time. We assume that a network should have at least a FIR $>10$. A FIR of 100 means less than 100 customers are hit at the same time, which can be considered rather safe for the operators. We believe that realistic networks should have a FIR between 10 and 100 .

\section{B. Scenarios}

We consider three scenarios for the reliability evaluation: dense urban (DU), urban (U) and rural (R). For the fiber availability, we have assumed a downtime of $\{0.5,0.3$ and 0.1$\}$ $\mathrm{hr} /(\mathrm{km}$-year) for DU, U and R, respectively. For the FF, we make a difference between the working path (WP) and backup path (BP) required for protected configurations (see section $\mathrm{V}$ ). A BP is only used for the FF since this is the most critical part for protection as will be shown in section IV.

The typical fiber length of the WP and BP in the three scenarios varies and depends on the degree of node consolidation. Node consolidation is the replacement of a number of active network sites (COs) with a CAN. For our calculations we have considered two node consolidation scenarios: High $(\mathrm{H})$ and Low (L). For high and low node consolidation, we have assumed a CAN replaces 80 and $4 \mathrm{COs,}$ respectively. Table 1 shows the fiber lengths for the different scenarios, as calculated in [7] for a household penetration of

Table 1: Fiber lengths for the considered scenarios

\begin{tabular}{|c|c|c|c|c|c|c|c|}
\hline \multicolumn{2}{|c|}{ Node Consolidation } & \multicolumn{3}{c|}{$\mathrm{H}$} & \multicolumn{3}{c|}{$\mathrm{L}$} \\
\hline \multicolumn{2}{|c|}{ Scenarios } & DU & $\mathrm{U}$ & $\mathrm{R}$ & $\mathrm{DU}$ & $\mathrm{U}$ & $\mathrm{R}$ \\
\hline \multicolumn{2}{|c|}{ DF+LMF length $(\mathrm{km})$} & 1.5 & 2.5 & 3.5 & 1.5 & 2.5 & 3.5 \\
\hline \multirow{2}{*}{ FF length $(\mathrm{km})$} & $\mathrm{WP}$ & 6 & 23 & 40 & 1 & 4 & 9.5 \\
\cline { 2 - 9 } & $\mathrm{BP}$ & 11 & 38 & 72 & 3.5 & 12 & 28 \\
\hline
\end{tabular}

$100 \%$. We have assumed a WP fiber length from the OLT to the ONUs between 2.5 and $7.5 \mathrm{~km}$ for DU, between 6.5 and $25.5 \mathrm{~km}$ for $\mathrm{U}$, and between 13 and $43.5 \mathrm{~km}$ for R. The fiber length of the BP will be larger than the WP due to the fact that the BP fiber will be laid in a disjoint duct.

\section{RELIABILITY PERFORMANCE EVALUATION OF

UNPROTECTED ARCHITECTURES: NEED OF RESILIENCE

In this section, we estimate the reliability performance of the various unprotected hybrid WDM/TDM PON architectures and identify where resilience is really required.

Fig. 2 gives the unavailability of various hybrid WDM/ TDM architectural elements. After a reach of $1 \mathrm{~km}$, feeder fiber (FF), split between the three considered scenarios, is the most dominating part. The unavailability of an OLT and a wavelength switched RN1 is relatively high. For customer experience, the ONU reliability is also crucial. The wavelength selective RN1 has high unavailability as it always uses an active reach extender (RE). There is a step jump in the unavailability of RN1 of wavelength split due to the need to use a RE after $16 \mathrm{~km}$. The reliability of the remaining elements is not crucial. This evaluation helps us to identify that FF, OLT, wavelength switched RN1, ONU and RN1 with REs are the real bottlenecks to reach the satisfied connection availability.

Fig. 3 gives the FIR values for different elements of the considered hybrid WDM/TDM PON variants. Note that we do not show the FIR of DF and LMF as it is expected to be quite



Figure 2. Unavailability of various unprotected architectural elements

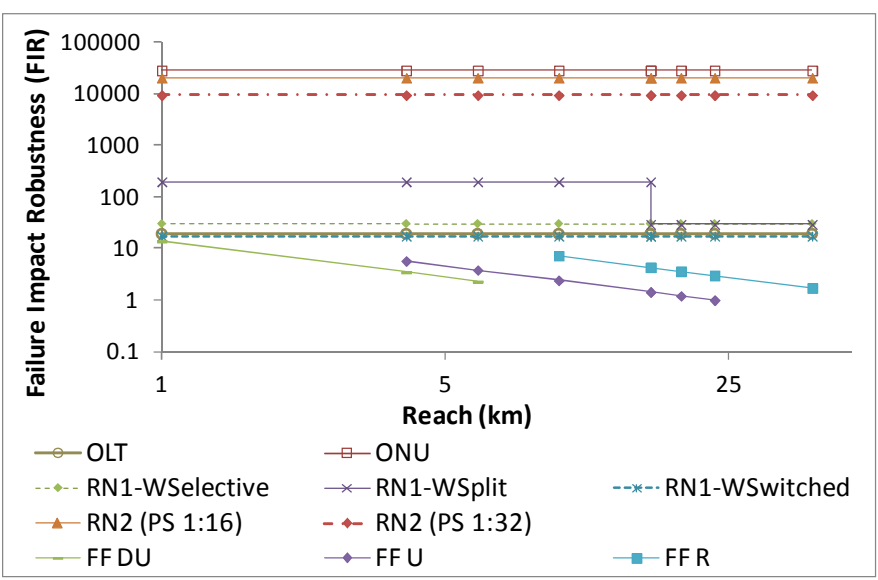

Figure 3. FIR of various unprotected architectural elements 
high due to the small number of end users affected by a failure occurred at DF or LMF. FF, OLT, wavelength switched RN1, and RN1s with REs are most important to protect. We see that from an operator perspective, it is not significant to protect an individual customer. It is most relevant to protect up to RN1 and not afterwards as there is no major failure impact of RN2 and ONU.

\section{RELIABLE ARCHITECTURES}

In this section, we discuss different protection options and then propose reliable architectures. The reliability evaluation has been done with respect to different population areas.

\section{A. Protection Scenarios}

\section{1) Protection at the $C A N$}

As shown in Fig. 3, the FIR for the unprotected OLT has the lowest value after the feeder fibers (FFs), which keeps the EtoE FIR of any configuration below an acceptable threshold. Therefore, it is crucial to provide protection at the CAN, where the OLT is located. Note that we have considered different OLT location which leads to independent power failures at the CAN improving reliability. OLT protection can be done either by duplicating the line terminal (LT) or by full OLT duplication [1]. Protecting the LT itself does not show much benefit to improve the unavailability and FIR of the OLT. It is because of the low availability of the active components such as the switch, power supplies, booster/preamplifier. Therefore, we consider full OLT duplication in this paper. Full OLT duplication needs inter-OLT signaling to control the switching for protection. There are two ways of connecting the duplicated OLTs to the WP and BP FFs. One is to use an extra $2 \times 23 \mathrm{~dB}$ coupler to combine the OLTs and FFs. The other is to connect each OLT output directly to each FF. In this paper, we consider the latter scheme which does not need an additional coupler and has a higher connection availability and FIR.

\section{2) Protection between the CAN and LE}

Protection between the CAN and LE is most significant for long FF. From an operator perspective, as soon as the FF length becomes more than $1 \mathrm{~km}$, the FF becomes the most dominant factor which influences reliability (see Fig. 3). Normally, FF protection duplicates the fiber, and as mentioned in section III, the BP FF is laid in a disjoint duct with minimal geographical overlap. Moreover, a FF cut affects the large customer base and hence impacts the FIR.

\section{3) Protection between the LE and end-user}

As it is already clear that the protection between the LE and end-users does not significantly affect the FIR, the network operators will generally be not in favor of protection after the LE. Note that for business customers or some services requiring high reliability such as e-health services providing remote consultation of doctors, end-to-end customer protection is required. In this paper, we focus on the general case and will not discuss the protection between the LE and end-users.

\section{B. Protected Architecture Designs}

In the previous sections, we have identified that protection at the CAN and the FF should be provided in order to have a satisfied connection availability and FIR for the end-users and operators. Moreover, when introducing protection for hybrid
WDM/TDM PON, the performance degradation in the other aspects, such as reach, supported number of end-users per FF and flexibility on resource allocation, should be avoided. With this in mind, we propose two protected architectures down to the LE (RN1) while keeping the performance degradation at a minimum level (see Fig. 4).

Both approaches in Fig. 4 have an identical protection scheme down to RN1 where the OLT and FF are duplicated. In the protected architecture shown in Fig. 4(a) the working and backup FFs are directly connected to one 2:M [8] device at RN1. As described in Section II, in basic hybrid WDM/TDM PON architectures, the device(s) at RN1 can be either a splitter, an AWG, or a combination of WSSs and AWGs according to the type of the hybrid WDM/TDM PON. In the second protection approach (see Fig. 4(b)), one $3 \mathrm{~dB}$ splitter is used for connecting the working and backup FFs to RN1. In this case duplicated devices are required at RN1 and the number of splitters located at RN2 needs to be doubled with half of the output port count (i.e. 1:N/2) compared to the scheme in Fig. 4(a). If needed, two REs can be placed at the end of each $\mathrm{FF}$, right before any other component at RN1. The two proposed protection schemes do not affect the maximal number of supported users per FF, flexibility on resource allocation and power budget for the connection between the OLT and ONU.

\section{Architectural Evaluation}

In this section, we evaluate the resilience performance of various architectures. Note that the proposed architectures may also differ in cost and power consumption but this is out of the scope of this paper. For a cost evaluation, we refer to [9]. Fig. 5 and Fig. 6 show that the protected OLT has a much higher availability and FIR than other RN1 elements. It is clear that without RE, RN1 has a much higher availability and FIR. Fig. 7 and Fig. 8 depict the availability and FIR of the three considered hybrid WDM/TDM PON variants for three scenarios (DU, U and L) with high $(\mathrm{H})$ and low (L) node consolidation. The maximum reaches for the wavelength selective, wavelength split and wavelength switched variants are equal to 30,75 and $39 \mathrm{~km}$, respectively. Note that it is not

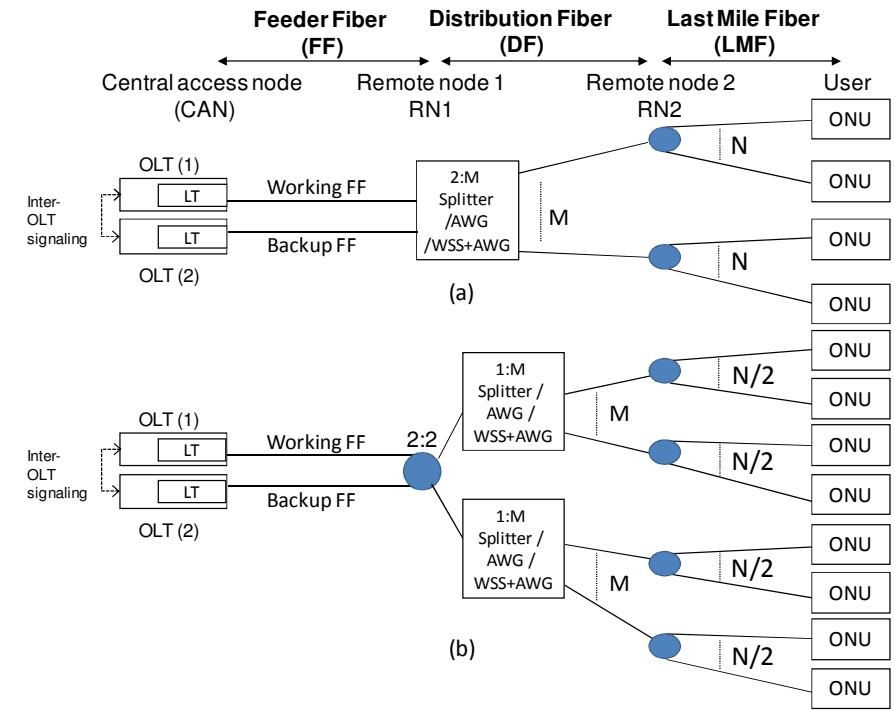

Figure 4. Two proposed reliable architectures for all types of hybrid WDM/TDM PON (a) without $3 \mathrm{~dB}$ splitter (b) with $3 \mathrm{~dB}$ splitter 


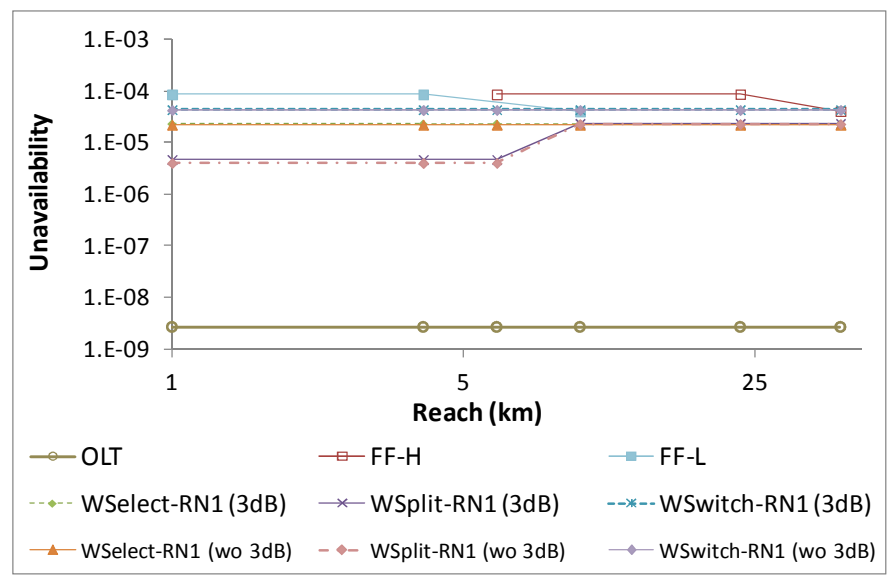

Figure 5. Unavailability of various protected architectural elements

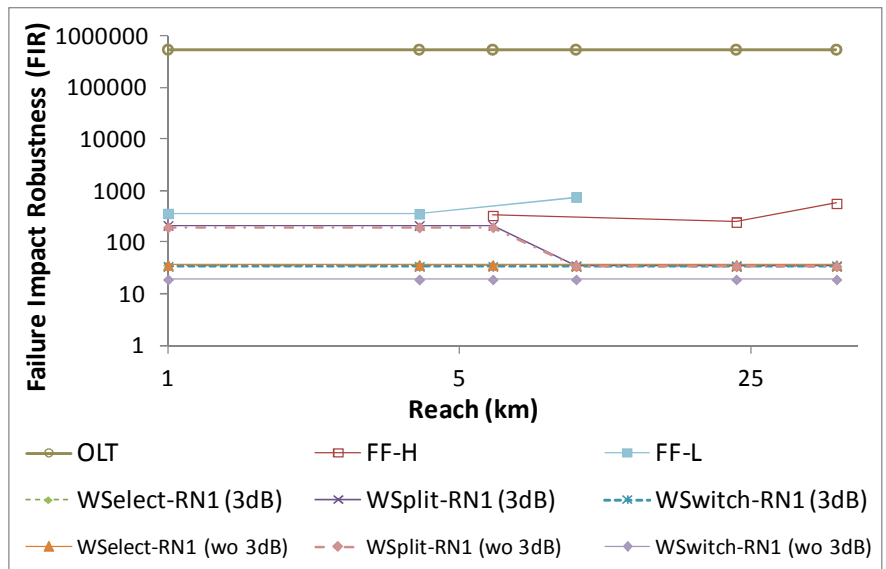

Figure 6. FIR of various protected architectural elements

possible for all variants to have $100 \%$ penetration in the high node consolidation scenario due to their reach limitation. Fig. 7 and Fig. 8 show a significant gain on the reliability performance of the two proposed protection schemes, namely with and without a $3 \mathrm{~dB}$ splitter, compared to the unprotected ones (UnPr). Protection with a $3 \mathrm{~dB}$ splitter achieves a higher FIR for the wavelength split and switched configuration. Generally, all protected scenarios achieve a FIR more than 10 and an availability close to 4 nines. The wavelength switched configuration has the lowest reliability amongst the three variants due to the use of a WSS at RN1. For small reach cases, wavelength split has a considerably higher reliability than a wavelength selective configuration as it does not require a RE. For long reach cases, however, it has the same or even a lower reliability.

\section{CONCLUSIONS}

In this paper, we have identified the most important elements for protection and proposed two efficient protection schemes for all three considered variants of hybrid WDM/TDM PONs. The reliability performance results have shown that from an operator perspective, protection beyond the first remote node (RN1) does not provide important gains as it can be achieved by FF and CAN protection. Moreover, our proposed protection schemes can significantly improve the reliability performance in terms of both connection availability and failure impact robustness while avoiding the performance degradation on the other aspects. For future work, protecting

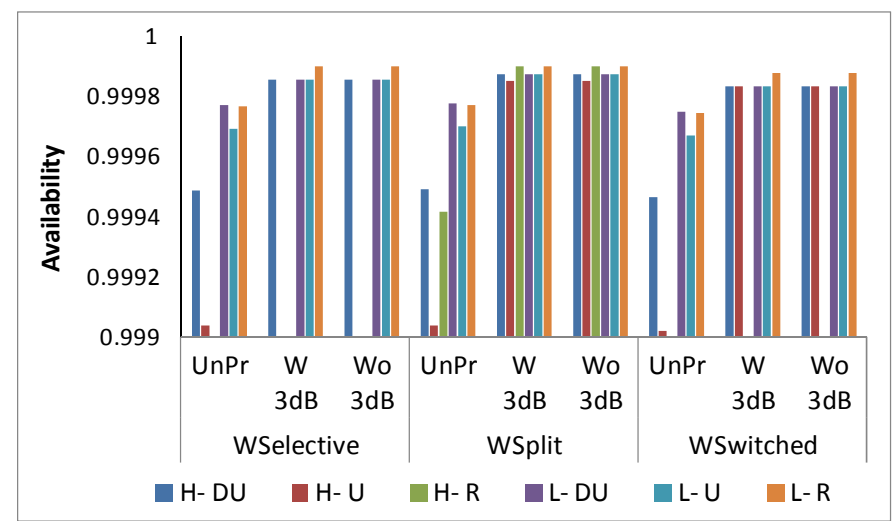

Figure 7. Availability of various unprotected and protected architectures

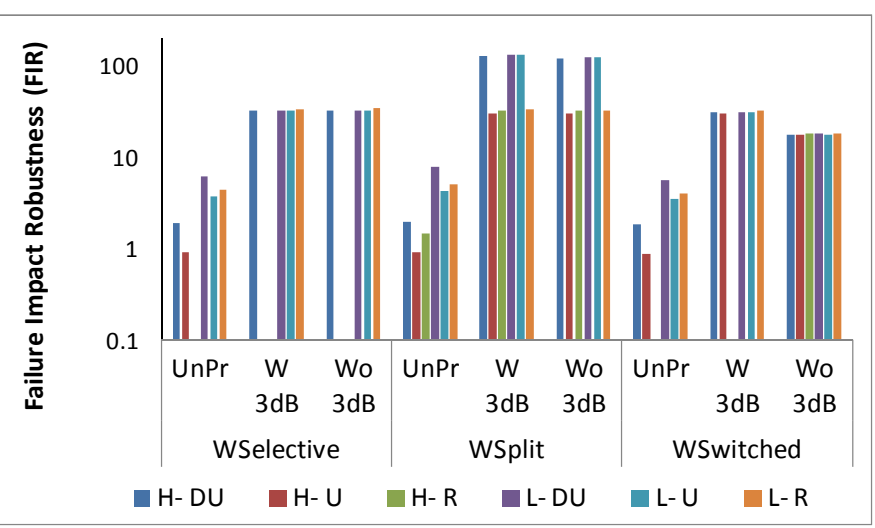

Figure 8 . FIR of various unprotected and protected architectures

the WSS-based RN1 can further improve the reliability for wavelength switched hybrid WDM/TDM PON.

\section{ACKNOWLEDGMENT}

The research leading to these results has received funding from the European Community's Seventh Framework Programme (FP7/2007-2013) under grant agreement $n^{\circ} 249025$ (ICT-OASE).

\section{REFERENCES}

[1] K. Grobe, and J.P. Elbers, "PON in adolescence: from TDMA to WDM PON", IEEE Communications Magazine, vol. 46, Jan. 2008, pp.26-34.

[2] OASE Project, D4.2.1: Technical Assessment and Comparison of NextGeneration Optical Access System Concepts, Oct. 2011.

[3] A. Dixit et al., "Flexibility Evaluation of Hybrid WDM/TDM PONs", IEEE ANTS 2011, Bangalore, India, Dec. 18-21, 2011.

[4] S. Verbrugge, D. Colle, P. Demeester, R. Huelsermann, and M.Jaeger, "General availability model for multilayer transport networks", DRCN 2005, Albergo della Regina Isabella, Italy, Oct. 16-19, 2005, pp. 85-92.

[5] J. Kim, S. Yen, S. Fu, and L. G. Kazovsky, "Resilient Optical Access Networks: Optimization on the number of Spikes in the StarRing", Workshop on Photonic Technologies for Access and Bio-Photonics, Jan. 2011.

[6] M. Vogt, R. Martens, and T. Andvaag, "Availability modeling of services in IP networks", DRCN 2003, Banff, Canada, Oct. 19-22, 2003, pp. 167-172.

[7] OASE Project, D5.1: Overview of methods and tools, Sep. 2010.

[8] Y. Akiyama, T. Terahara, and H. Ooi, “ Optical cross-connect using wavelength selective switches", US Patent 7933 519, Apr. 26, 2011.

[9] B. Lannoo et al., "Techno-economic feasibility study of different WDM/TDM PON architectures", ICTON 2010, Munich, Germany, Jun. 27 - Jul. 1, 2010. 